\title{
Leopardi, la caduta, il sapere e la vanità della vita
}

Anche se nell'agosto del 1820 Leopardi ama credere che "le illusioni ... durano ancora a dispetto della ragione e del sapere," teme tuttavia che "l'incremento e divulgazione della filosofia" distruggano "tutte le illusioni" e che, di conseguenza, "di questa razza umana non resteranno altro che le ossa" (Zibaldone I 223). Il poeta sa che la natura, saggia ordinatrice dell'universo, conosce il pericolo del sapere, e che ad esso s'oppone volendo il vigore del corpo e l'uomo libero dal pensare. "Il vigore del corpo nuoce alle facoltà intellettuali, c favorisce le immaginative." Gli antichi ubbidivano alla natura, e "si procacciavano il vigore del corpo" per "mantenere il vigore dell'animo" e "le illusioni." Ma i moderni, diversi dagli antichi, ignorando che "l'imbecillità del corpo è favorevolissima al riflettere," trascurano il vigore fisico, pensano molto e le "grandi illusioni" sono loro sconosciute (Zibaldone 140-41). Il giudizio di Lcopardi sul sapere incupisce con il passar del tempo. "Se molti sapienti hanno conosciuto la tristezza e la vanità delle cose," Leopardi scrive al pesarese Giulio Perticari nel 1821, "io . . ho conosciuto anche la tristezza e vanità della sapienza” (Lettere 314). Nel 1823 la fclicità, un tema che appassiona il poeta nello Zibaldone, gli appare negata dal sapere. Medita sulla corruzione e decadenza del genere umano c conclude che esse nacquero "dal sapere, e dal troppo conoscere" (Zibaldone II 180-81). E alcune settimane avanti Leopardi aveva già visto che la vera sapienza è libertà dal sapere: "Sapientissimi" sono il fanciullo non tocco dall'educazione e "il sclvaggio della California, che non conosce il pensare" (Zibaldone II 57).

Cos'è questo sapere contro il quale Leopardi inveisce e quali erano gli effetti ch'egli paventava? Perché si scaglia contro il sapere, la cosa alla quale dedicò le sue energie e la sua vita? Come nasce l'ostilità contro il sapere? Per ottenere qualche risposta occorre risalire alla peculiare interpretazione leopardiana della caduta contenuta nelle lunghe riflessioni sul Cristianesimo del dicembre 1820. Qui 
per la prima volta nell'opera del poeta insorge in modo organico l'ostilità contro la sapienza. Leopardi crede che Dio comandò ad Adamo ed Eva di astenersi dai frutti dell'albero nel Paradiso terrestre per impedire loro di acquistare il sapere. Egli cita Genesi, II, 17 , in latino ("De ligno autem scientiae boni et mali ne comedas, in quocumque enim die comederis ex eo morte morieris") e commenta: "Non è questo un interdir chiaramente all'uomo il sapere? Un voler porre sopra tutte le altre cose ... un ostacolo agl'incrementi della ragione. .. ?" (Zibaldone I 337). Per Leopardi l'importanza della caduta consiste, non nell'offesa arrecata all'autorità divina, non nella degradazione subita da Adamo ed Eva, e non nell'acquisto della scienza del bene e del male. Consiste invece nell'atto cognitivo implicito nella violazione del comandamento, nel sapere: Adamo ed Eva mangiarono il frutto proibito e seppero d'essere nudi: "APERTI SUNT OCULI AMBORUM, cumque COGNOVISSENT se esse nudos" (Zibaldone I 339). ${ }^{2}$ E Leopardi precisa: "Dunque l'aprir gli occhi, dunque il conoscere fu lo stesso che decadere o corrompersi, dunque questa decadenza fu decadenza . . non di ragione o di cognizione." Al contrario, la caduta incrementò il sapere, e "l'uomo seppe quello che prima non sapeva . . . cioè di esser nudo." Con la caduta l'uomo commise un duplice peccato: fu incapace di superare l'ostacolo e il pericolo "della ragione e del sapere" che s'opponevano alla felicità perenne, e volle sapere piú di quel "che gli conveniva sapere . . . per opera sua, cioè non piú per natura, ma per ragione" (Zibaldone I $338,339,340)$.

Intanto l'uomo "restò piú sapiente di quando era stato creato" e, a causa della sua accresciuta sapienza, "veramente simile a Dio" ( $\mathrm{Zi}$ baldone I 339). Simile a Dio, ma non Dio, e insoddisfatto della sua pur grande sapienza. Quindi, roso dal desidero di divenire migliore di quel che era, di perfezionarsi e di uguagliarsi a Dio. Sorge cosí l'aspirazione alla "perfettibilità," l'aspirazione che implicitamente sanziona la nullità della vita. Come? Perché "sostenendo la perfettibilità dell'uomo" si sostiene che "l'opera della natura, cioc̀ di Dio, era imperfetta; che l'uomo può essere perfezionato non già da Dio, ma da se stesso" (Zibaldone I 336), che quando l'uomo avrà percorso il sentiero della perfettibilità e ne avrà raggiunto il fine, la sua esistenza sarà divenuta perfetta, sarà cioè migliorata immensamente rispetto all'attuale. Questo significa che la vita attuale non solo è 
immensamente inferiore a quella immaginaria, futura e perfetta, ma che nello sforzo continuo richiesto dal raggiungimento della perfettibilità, la vita cosí com'è va anche superata e ripudiata ad ogni suo stadio, significa che la vita attuale non ha alcun valore.

La "Storia del genere umano," la prima delle Operette morali composta tre anni dopo le meditazioni fatte nello Zibaldone, dà una buona idea della perfettibilità e del suo rapporto con la nullità della vita. Immuni da "pazze idee d'incremento, di perfezioni" nell'età giovanile, i primi uomini del genere umano traevano "da ciascun sentimento della loro vita incredibili diletti" e "crescevano con molto contento." Ma nell'età matura divennero scontenti del creato e di se stessi. Vollero allora accrescere il bene che già godevano perché, "contentarsi di quello che presentemente godessero, senza promettersi verun accrescimento di bene, non pareva loro di potere." La scontentezza si tramutò in odio per la vita, e persino indusse alcuni uomini al suicidio. Giove, allarmato, s'adoperò per sollevare la sorte dei mortali; abbellí il creato e lo rese piú vario. La vita dei primi uomini migliorò, ma per poco tempo, chè la scontentezza ben presto li riprese e diffuse tra loro la "disistima della vita." Giove allora seppe che gli uomini, non importa in quale stato essi siano, bramano "l'impossibile" e "si travagliano con questo desiderio." Egli, nondimeno, migliorò ancora una volta la vita degli uomini disseminando fra loro il male affinché essi godessero ogni qualvolta riuscivano a liberarsene. L'intento fu raggiunto, ma solo temporaneamente. Gli uomini furono ripresi dal "fastidio delle cose loro" e "dall'amaro desiderio" per un bene tanto superiore quanto irraggiungibile. E quando piú tardi Giove avrà inviato tra gli uomini la "Sapienza" e la "Verità," allora "Saranno stati ritolti alla terra i suoi fantasmi, c . . mancherà dalla vita umana ogni valore, ogni rettitudine, cosí di pensieri come di fatti" (Operette morali I 811-18, 821). La corsa alla perfettibilità gencra il tormento mai alleviato di raggiungere una meta che, essendo impossibile da conseguire, produce il risultato opposto alla perfezione-infelicità e svalutazione della vita.

L'idea della perfettibilità nata dal sapere inquieta Leopardi, e già nelle sue meditazioni del dicembre 1820 , pur sfuggendogliene tutta la portata, ne intuiva l'ingannevolezza ed arroganza. Incolpava $i$ sostenitori della perfettibilità dell'uomo di superbia e i primi padri d'aver peccato "per aver sognata questa perfettibilità, e cercata questa 
perfezione fittizia." E precisava che l'accresciuto potere cognitivo li rese superbi: "La loro superbia non consiste in altro che nella ragione: ragione assoluta" (Zibaldone I 338-339). Ma nelle meditazioni del 1820 un'altra conseguenza della caduta e del sapere-l'immortalità-inquieta il poeta ben piú della perfettibilità. L'immortalità, la credenza nella "felicità di un'altra vita" e nell' "infelicità di questa vita," insidia il valore di questa vita piú della perfettibilità. Siccome con la caduta "la ragione aveva preso il disopra sulla natura ... l'uomo era divenuto infelice" ed egli era condannato "all'infelicità della corruzione." Ma Dio, nella sua infinita misericordia, volle dare all'uomo "una perfezione compatibile colla sua condanna, cioè colla sua infelicità" e decise di "perfezionare la sua ragione, cioè quella parte che aveva prevaluto immutabilmente nell'uomo per la sua disubbidienza." Ora, "la perfezione della ragione consiste in conoscere la sua propria insufficienza e felicitarci, anzi l'opposizione intrinseca ch'ella ha colla nostra felicità" e non può che "condurre . . . alla felicità di un'altra vita." La ragione fece capire all'uomo due cose: che dopo la caduta "non poteva essere la sua felicità in questa vita," e che poteva soltanto concepire e credere nella "sua felicità in un'altra vita." Insomma, "col prevaler della ragione e del sapere l'uomo, non potendo piú credere quello che credeva naturalmente" prima del peccato originale, cioè ch'egli era felice cosí com'era, sentì il prepotente bisogno di credere che c'era ancora felicità per lui, e tornò e crederlo "mediante questa medesima ragione e questo sapere che non si poteva piú estinguere." E lo credette concependo un'altra vita in cui avrebbe goduto la felicità di cui era stato privato in terra $(Z$, I, 342-44, 348).

Avendo poi "la Religione ... divinizzato la ragione e il sapere," essa ha anche codificato l'infelicità di questa vita ed esaltato la felicità dell'altra. Cosí il "Cristianesimo chiama beato chi piange, predica i patimenti, li rende utili e necessari; in una parola suppone essenzialmente l'infelicità di questa vita." La dottrina cristiana ha fatto sua la conseguenza ultima della caduta e del trionfo del sapere: la vanità della vita. La dottrina insegna al fedele di vivere, sí, sulla terra, ma solo per "indirizzar questa ad un'altra vita, rispetto alla quale solamente, è ragionevole questa vita: e che questa sarebbe necessariamente infelice." Sotto il Cristianesimo infatti "la vita ... fu . . molto minore, meno attiva, meno bella, meno varia, e 
precisamente piú infelice" che Ira gli antichi. I poemi di Ossian, per esempio, mostrano quanto gli antichi Celti, non tocchi dal Cristianesimo, fossero "Iontani dal concepire la nullità . . . della vita." E prima dell'avvento del Cristianesimo, "la disperazione e scoraggiamento della vita . . l'odio della vita come vita umana . . la nullità e noia inerente ed essenziale alla nostra vita, in somma l'idea che la vita nostra per se stessa non sia un bene, ma un peso e un male, non è mai entrata in intelletto antico." Non è mai entrata in mente antica perché il Cristianesimo era sconosciuto all'antichità. Ma sotto il Cristianesimo, l'uomo "non ha cura se di una patria situata nell"altro mondo," ed egli allor "considera questa terra come un esilio." Abituato "alla speranza di beni d'un'altra vita," egli diviene inetto per questa e incapace di sentire "quei grandi stimoli che producono le grandi azioni." Il dogma cristiano dell'immortalità ha cosí "contribuito non poco a distruggere il bello il grande il vivo il vario di questo mondo, riducendo gli uomini dall'operare al pensare e al pregare" (Zibaldone I 248-342, 343, 357, 389). E l'anno seguente, il 1821, Leopardi insiste ancora che il Cristianesimo "è incompatibile ... con la sussistenza del mondo e della vita umana" perché "surrogando un altro mondo al presente . . . viene . . . a distruggere il mondo" ed induce il cristiano a tener "se stesso per un nulla" (Zibaldone I 951, 1092). Nell'intelletto cristiano campeggia il comando "di fuggire la vita ... schivare la vita . . annullare quanto è possibile l'esistenza . . . fare che l'esistenza non s'impicghi ad altro che a premunirsi contro l'esistenza" (Zibaldone I 1435. 1436). Il pensiero e l'atteggiamento di una non meglio identificata madre di famiglia, ma quasi certamente quella di Lcopardi stesso, Adelaide Antici, rispecchia fedelmente la negazione della vita voluta dal Cristianesimo. Questa madre, "saldissima ed esattissima nella credenza cristiana e negli esercizi della religione," considerava la bellezza c il rigoglio della gioventú come una vera disgrazia. Essa ringraziava Dio perché le aveva dato figli brutti e deformi, e pretendeva che a causa della deformità (di cui, guarda caso, Giacomo era un raro esemplare), "rinunziassero inticramente alla vita nella loro prima gioventù (Zibaldone $1309-310$ ).

Un breve scritto, Comparazione delle sentenze di Bruto Minore e di Teofrasto vicini a Morte (1822), lumeggia il rapporto tra il sapere, la nullità della vita e la sete d'immortalità che Leopardi andava 
forgiando. Quando il filosofo greco arrivò "a conoscere la somma della sapienza," capí allora la "miseria che nasce dalla perfezione e sommità della sapienza" e "la vanità della vita e della sapienza" stessa. Riesce incredibile, nota Leopardi, che il "sentimento della vanità della vita" albergasse nell'animo di Teofrasto, perché i suoi contemporanei, gli antichi, credevano nel valore e nella felicità della vita. Onde spiegarsi l'incredibile nichilismo di Teofrasto, Leopardi argomenta che il greco soccombette al sentimento della vanità della vita perché mise la sapienza al servizio della verità e scoprí quello che non avrebbe mai dovuto scoprire-“l'inutilità dei sudori umani." Imparata la triste lezione, Teofrasto morí ammonendo i suoi discepoli contro tutto ciò a cui egli aveva dedicato la vita-lo studio e il sapere-e rammentando loro che la "vanità della vita è maggiore che l'utilità" (Comparazione 1045).

Un critico nota che con la storia della crisi intellettuale di Teofrasto, Leopardi scopre il pessimismo tra gli antichi e lo fa suo (Timpanaro 199, 200, 203). Ma Leopardi non avrebbe scoperto questo pessimismo, questa coscienza della nullità della vita tra gli antichi, senza il frutto tratto dalle meditazioni fatte sulla caduta, sul sapere e sulla vanità della vita nel dicembre 1820 . È vero che Teofrasto, morto nel 285 A.C., arrivò al suo nichilismo senza sentire il peso del peccato originale e senza l'impulso dell'insegnamento cristiano. Ma il sapere produsse su Teofrasto lo stesso effetto ch'esso produsse su Adamo-il sentimento della vanità della vita. E quando Giove nella "Storia del genere umano" manderà tra gli uomini ancora pagani, la "Sapienza" e la "Verità," l'accresciuto sapere produrrà ancora lo stesso risultato. Piú di due secoli separavano l'età di Teofrasto da quella del Cristianesimo e il filosofo non sentí neppure la sete dell'immortalità. Ma nella Comparazione questa sete appare nondimeno, e i contemporanei di Bruto (85-42 A.C.), ormai sulla soglia dell'era cristiana, la sentono in tutta la sua forza. Questi non seppero liberarsi da quel sentimento della vanità della vita sorto nell'età di Teofrasto e, non volendo suicidarsi come Bruto, s'arresero alla potente ma fatale illusione della dottrina cristiana, di quella dottrina che doveva di lí a poco spargersi tra il mondo antico in sfacelo. "Mancato ogni pregio a questa vita," scrive Leopardi nell'ultima pagina della Comparazione, "cercavano i sapienti quel che gli avesse a consolare ... della vita medesima, non riputando per credibile che 
l'uomo nascesse . . alla miseria. Cosí ricorrevano alla credenza e all'aspettativa d'un'altra vita, nella quale stesse quella ragione della virtú e de' fatti magnanimi, che ben s'era trovata fino a quell'ora, ma già non si trovava e non s'aveva a trovare mai piú, nelle cose di questa terra" (Comparazione 1045).

Alla perfettibilità e all'immortalità, a queste conseguenze della caduta e del sapere, Lcopardi ritornerà a piú riprese nel corso degli anni per stimarne gli effetti sul genere umano e per ripudiarle. S'avvede che per raggiunger la perfezione il genere umano deve percorrere una lunga e disagevole via; deve "stentare, tentare mille strade, sbagliare mille volte, e tornare indietro, e finalmente . . aspettare lunghissimo ordine di secoli per conseguire in parte il detto fine." Ma è ragionevole supporre che dopo tanti sforzi e tante delusioni l'umanità si ritroverà finalmente felice? Non c̀ invece da temere che dopo tanti travagli essa avrà esaurito ogni possibilità di condurre una vita migliore, perfetta? È cosa certa che l'umanità pur avendo fatto prove e patito sofferenze per lunghissimo tempo, non è ancor riuscita a raggiungere la prefissa meta. Di che giovamento sarà questa perfezione, "posta ... au bout di sí lunga e difficile carriera, che dopo seimila anni ancora non è compiuta?" Essendo impossibile intravedere quando l'agognata perfezione sarà divenuta realtà, quando cioè i travagli del genere umano avranno fine, c'è ragione di disperare. "Quando e come saremo noi perfetti, cioè veri uomini? In che punto, in che cosa consisterà la perfezione umana? Qual sarà la sua essenza?" si chiede esasperato il poeta nel settembre 1821. Poiché l'essenza della perfezione ci è sconosciuta, è impossibile definire il punto in cui l'uomo sarà perfetto. Qual grado di sviluppo deve ritenersi lo stato di perfezione al quale il genere umano era destinato? Il presente o un altro? Se un altro, quale? È impossibile saperlo. Il presente, o ogni altro grado dello sviluppo è per quanto ci è dato sapere, niente altro che "una delle diecimila diversissime condizioni a cui potevamo ridurci” (Zibaldone I 564, 1028, 1051-52).

All'uomo fu assegnato un corpo la cui composizione e funzionamento sono quanto di piú perfetto si possa immaginare, un organismo che, Leopardi pensa, difficilmente può esser migliorato. Ora, l'idea della perfettibilità presuppone innanzitutto la perfezione dello spirito, un'entità molto piú complessa del corpo, molto piú difficile da capire e da modificare. Come possono, Leopardi si chiede, preten- 
dere i sostenitori della perfettibilità che lo spirito sia portato ad un ipotetico grado di perfezione se l'uomo non sa neppure perfezionare la parte meno complessa del suo essere, il corpo? Quel poco di progresso fatto finora dalla civiltà, i passi fatti verso la cosidetta perfezione, hanno in realtà causato un allontamento dalla perfezione. "Giacché è incontrastabile che questa pretesa perfezione dell'animo nuoce al corpo," la maggior attività intellettuale e spirituale che ha accompagnato la civiltà ha indebolito c reso inetto il corpo. Dunque "il perfezionamento dell'uomo include . . . il corrispondente e sempre proporzionato deterioramento e . . imperfezionamento di una piccola parte di esso uomo, cioè del suo corpo." Lo squilibrio tra intelletto c corpo non era predisposto dalla natura perché in essa tutto "è armonia, ma sopratutto niente in essa è contraddizione." Una perfezione che presuppone il deterioramento fisico è un'assurdità, la negazione stessa di ogni idea di perfezione (Zibaldone I 322, 1043; II, 307). ${ }^{4}$

La critica dell'idea della perfettibilità si rinnova vigorosa nel 1821 e conduce alla rivalutazione della vita come è nella sua dura realtà. "La perfezione assoluta," Leopardi scrive nello Zibaldone, "e l'esistenza, sono termini contraddittorii." Riconosciuto che le idee innate, non esistono, l'idea della perfettibilità vien meno perché essa, come le idee innate, non è "indipendente dalla cose quali elle sono." La perfettibilità è fomentata dalla mentalità volta a negare l'attualità spiacevole della vita in nome del pregiudizio che vuole la perfezione fuori del reale, che la concepisce estranea alle cose create. L'esistente, Leopardi risponde, va riconosciuto "tutto per relativo, c relativamente vero." Rinunciamo, il poeta propone, "alle pazze idee d'incremento," accettiamo l'idea che a questo mondo "nulla è perfetto in un modo che non è, in un modo in cui le cose non sono," sostituiamo all'idea di perfezione assoluta quella di perfezione "relativa." Si vedrà allora che l'autentica perfezione consiste in "quello stato che'è perfettamente conforme alla natura di ciascun genere di esseri" e si dovrà concludere che "l'uomo è perfetto qual egli è . . . e non può se non essere imperfetto in altro stato" (Zibaldone I, 1054-55, 1205-207). E quando nel 1824 nella "Scommessa di Prometeo," Momo e Prometeo si prefiggono di accertare se l'uomo sia "la piú perfetta creatura dell'universo," e scoprono che solo una minima parte del genere umano è progredita, che c'è voluta "quantità 
innumerabile di secoli" per compiere quel progresso, e che l'umanità è, tuttavia, ancora lontana dalla perfezione. Leopardi sa allora che la perfezione è una chimera e che l'imperfezione è il solo stato consonante al genere umano. Questo, il poeta conclude, è "veramente sommo tra i generi ... ma sommo nell'imperfezione, piuttosto che nella perfezione." E l'imperfezione, che è perfezione, include anche il male, anzi "tutti i mali possibili": la vita è perfetta cosí com'è posseduta dal male, da "tanto male quanto vi può capire" (Operette morali I 861, 865-66).

Quando il poeta acquista la piena coscienza che la pretesa "facoltà di perfezionarsi" è disgiunta dal "necessario" richiesto per attuare la perfezione, la sua ostilità si fa amaro sarcasmo. "Vi par questa una bella provvidenza," inveisce nel 1822 contro l'improvvida c crudele provvidenza, "dare all'uomo la facoltà di perfezionarsi," ma frattanto, poiché la "perfezione non si poteva conseguire se non dopo lunghissimo spazio di tempo, e successione d'infinite esperienze, fare decisamente, e deliberatamente infelici un grandissimo numero di generazioni," tutte quelle generazioni che si sono travagliate per raggiungere questa "difficilissima e remotissima" perfezione, questa perfezione circa la quale "non possiamo congetturar neppure in che cosa potrà consistere ... se mai s'otterrà” (Zibaldone I 1441, 1442). E nel 1824 l'ostilità lcopardiana si traduce nell'immaginaria punizione del genere umano il quale, avendo nella "Storia del genere umano" bramato la perfezione, incorre nell'ira di Giove. Gli uomini della prima operetta morale "si querelavano principalmente che le cose [del mondo] non fossero immense di grandezza, né infinite di beltà, di perfezione e di varietà." Si lagnavano che le cose crano "angustissime, tutte imperfette" c desideravano "infinita la perfezione e la felicità delle cose e degli uomini." Quando Giove capí che i mortali erano posseduti dal demonio di una perfezione irraggiungibile, deliberò "di punire in perpetuo la specie umana, condannandola per tutte le età future a miseria molto piú grave che le passate." Mandò tra gli uomoni la "Sapienza" c la "Verità" perché capissero che "per niuno accidente e niuno rimedio non la possano campare, né mai, vivendo, interrompere," c perché siano sempre tormentati dal "desiderio di un'immensa felicità" (Operette morali I 812, 813, $818,820,821$ ), di una felicità che, Leopardi insisterà ancora due anni dopo la composizione della "Storia del genere umano," deriva 
dalla "tendenza della vita dell'anima ad un fine impossibile a conseguirsi" (Zibaldone II 1014). La conclusione della "Storia del genere umano" riproduce su uno sfondo favoloso, il tragico risultato rappresentato dalla biblica storia di Adamo nello Zibaldone tre anni prima: la vittoria del sapere e l'inaridimento delle radici della vita.

La critica leopardiana dell'immortalità ha maggior forza di quella della perfettibilità. La ragione è duplice. Il poeta sa che la credenza nell'immortalità è piú diffusa tra gli uomini e piú radicata nel loro animo che non l'idea di perfettibilità. In secondo luogo, ha capito che questa credenza ripudia la vita terrena e che, implicitamente, consacra la nullità della vita. È una credenza tenace e pericolosa, e l'impegno di Leopardi nello stroncarla maggiore. Già qualche settimana dopo le meditazioni sul peccato originale, Leopardi verga nello Zibaldone tre pagine sull'anima improntate a crudo materialismo. S'iniziano con l'affermazione che "la mente nostra non può non solamente conoscere, ma neppur concepire alcuna cosa oltre i limiti della materia," s'appoggiano sul noto sillogismo che "se lo spirito non può perire per ciò che non si può sciogliere, cosí anche perché non si può comporre, non potrà cominciare," e si concludono nel convincimento che "non c'è ragione veruna perché l'anima ... supposta anche e non ostante l'immateralità, debba essere immortale" (Zibaldone I 450, 451, 452). L'anno seguente, il luglio 1822, il poeta definisce la materia il complesso delle "cose che influiscono" sull'uomo appena egli "entra nel mondo." E queste cose "sono le presenti, le sensibili, o quelle le cui immagini sono ... fomentate dalle cose in qualunque modo sensibili ad un altro tipo di cose- "le cose che ... appartengono ad uno stato di natura diversa dalla nostra presente, cioè al nostro stato dopo la morte"-e, ritornando all'idea dell'uomo immerso nelle cose sensibili, implicitamente nega l'al di là: "Vivendo noi fra la materia . . . le cose . . che . . . appartengono al nostro stato dopo la morte . . non hanno che far punto con niente di quello la cui esistenza sperimentiamo, e trattiamo, e sentiamo" (Zibaldone 1 1532).

A questo spassionato filosofare fa luogo nel 1822 una critica risentita. In luglio Leopardi compone l'Inno ai patriarchi. Qui la credenza nell'immortalità è giudicata perniciosa perché, inducendo l'uomo a pensare alla morte e a temerla, gl'infonde incertezza e dubbio sul suo stato futuro e gli avvelena la vita. La vera età dell'oro 
fu, non quella in cui l'uomo visse in pace e libero da cure mortali, ma quella in cui la stirpe umana visse senza conoscere il suo destino mortale e in cui s'avvicinava alla morte imperturbata. Allora Leopardi canta la felicità di coloro che, liberi dal pensiero dell'al di là, non conoscono il timore della morte: "Fra le vaste californie selve / nasce beata prole, a cui non sugge / pallida cura il petto" ed "inopinato il giorno / dell' atra morte incombe" (104-106, 109-110). I selvaggi californiani "veggono la morte. . ., ma non la preveggono," e questa è una delle ragioni per cui "l'occhio loro è allegro e vivace. . . : non alberga fra loro nè tristezza nè noia" (Argomenti 432). Nessun selvaggio, Leopardi scriverà ancora anni dopo la composizione dell'Inno ai patriarchi negando l'immortalità,

... alcun di premii o pene

Destinate agli spenti ebbe sentore,

Né già dopo il morir delle terrene

Membra l'alme credè viver di fuore. (Paralipomeni VIII: 12)

Nel settembre dell'anno seguente, il 1823, Leopardi ritorna all'asSalto dell'immortalità da un'altra direzione, quella della felicità. Siccome all'uomo riesce impossibile concepire quale sarà la felicità che l'attende nell'altra vita, né la felicità né l'altra vita, il poeta argomenta, esistono. Mentre "la felicità che l'uomo naturalmente desidera è una felicità temporale ... materiale, e da essere sperimentata dai sensi . . . una felicità insomma di questa vita," il Cristianesimo promette invece al fedele "un felicità grandissima e somma ed intiera.' ' Tanto grande e somma, ché l'uomo non la può né "comprendere né immaginare né pur concepire." Egli sa di certo però ch'essa è "di natura affatto diversa ed aliena da quella che in questo mondo si desidera." Ora il Cristianesimo promettendo all'uomo, infelice su questa terra, "una felicità celeste . . intera e infinita, e superiore senza paragone alla terrena," gli fa una burla crudele: "Si è come a una che si muor di fame e non può ottenere un tozzo di pane, preparagli un letto morbidissimo, o promettergli degli squisitissimi e beatissimi odori." Insomma, la credenza nella "felicità de' Beati" è per l'uomo tanto assurda quanto lo sarebbe la credenza nella felicità umana per la bestia, e la credenza nella felicità animale per la pianta (Zibaldone II 482, 483, 484-485).

Tra il 1826 e il 1827 il poeta lancia nella sua prosa gli ultimi due attacchi contro l'immortalità. Con il primo attacco, la dipinge come 
una credenza che avvelena la vita terrena; con il secondo, il poeta s'appoggia sull'istinto e sull'emozione per refutarla. Platone, dice Porfirio nel "Dialogo di Plotino e di Porfirio," ideò e sparse coi suoi scritti "quelle dottrine della vita avvenire accioché gli uomini, entrati in dubbio e in sospetto circa lo stato loro dopo la morte, per quella incertezza e per timore di pene e di calamità future, si ritenessero nella vita dal fare ingiustizia e dalle altre male opere." Platone mal riuscí a tener gli uomini lontani dal male; riuscí invece ad instillare negli uomini il "dubbio terribile" che la sorte dopo la morte ispira, e ad infondere quel dubbio in chi meno merita danno. La credenza e i dubbi sull'al di là spaventano "in sulle ore estreme," ma sgomentano "i buoni," coloro che "hanno volontà non di nuocere ma di giovare," piú dei malvagi. Sgomentano anche "le persone timide e deboli di corpo," aliene dalla violenza e dalle iniquità. Ma non sbigottiscono i malvagi, "coloro ai quali ... si richiederebbe altro freno che della sola legge," coloro che non si "tengono dal male operare." Le dottrine della vita avvenire fanno soffrire l'animo retto piú dell'iniquo, perché lo scrupolo dell'onestà impedirà al giusto di sentirsi e credersi "cosí netto e puro" come la severa legge cristiana gl'impone; non basterà insomma, "la coscienza della piú retta e travagliosa vita ad assicurare l'uomo in sull'ultimo ... dallo spavento dei castighi." E Porfirio conclude il "Dialogo" puntando un dito accusatore al filosofo greco: "Per le tue dottrine il timore . . . è fatto signore dell'uomo"; e il genere umano, già "esempio mirabile d'infelicità in questa vita," accrescerebbe la sua infelicità in questa vita," accrescerebbe la sua infelicità se credesse in quelle dottrine (Operette morali I 1003-1006).

Taluni suppongono, Leopardi scrive iniziando l'altro attacco, che l'immortalità esista come una specie di terra di nessuno dove i defunti vivono dapprima soli e sperduti, ma poi ottengono la consolazione di riunirsi agli amici e ai parenti quando saranno anch'essi morti. Noi sentiamo, il poeta medita ribattendo questa supposizione, istintiva tenerezza e dolore per i morti, "gli stimiamo infelici . . . tenghiamo per misero il loro caso, e la morte per una sciagura." Allora, "se l'uomo è immortale, perché i morti si piangono? Perché aver compassione ai morti, perché stimarli infelici, se gli animi sono immortali?" La risposta è semplice: sprigiona dalla convinzione che il defunto è scomparso, che "la vita e l'essere" sono stati distrutti, che "i morti 
sieno morti veramente e non vivi; e che colui ch'è morto, non sia piú. I superstiti si addolorano perché il trapassato "ora non vive e non è." Duole loro che egli "abbia sofferta quest'ultima c irreparabile disgrazia," di essere scomparso per sempre. Siccome il pensiero di una vita eterna in compagnia dell'anima di una persona cara non ha mai consolato alcuno si deve concludere che quando la morte priva gli uomini di coloro che sono loro vicini, "nel fondo del loro cuore, piuttosto consentono in credere la estinzione totale dell'uomo, che la immortalità dell'animo" (Zibaldone II 1111-1113).

Le considerazioni sull'immortalità culminano in quel torno di tempo, il 1826-1827, in un rovente attacco contro lo spirito, il grande protagonista della vita immortale e contro il sapere, l'origine della credenza nell'immortalità. Riprendendo l'idea fissata nel febbraio 1821 sulla materialità dell'essere, il poeta definisce lo spirito "sostanza che non è materia" e quindi "sostanza che non è di quelle che noi conosciamo o possiamo conoscere o concepire." Contrappone poi la sua idea a quella spiritualista, riconosce la diffusione e il predominio di questa, stupisce e s'abbatte costatando che "questo spirito ... c̀ stato per lunghissimo spazio di secoli creduto contenere in sé tutta la realtà delle cose; e la materia . . è stata creduta non essere altro che apparenza, sogno, vanità appetto alla spirito." Deplorabile "miseria dell'intelletto umano" preso da siffatto "delirio"! Ma, peggio ancora, gli uomini delirano piú nel secolo decimono che nel passato; in questo secolo "lo spiritualismo" impera persino tra le piú progredite nazioni europee e i loro piú illuminati filosofi si compiacciono di riconoscere il trionfo dello spirito. "O Verità," Leopardi esclama davanti a questa catastrofe della civiltà moderna, "tu sei sparita dalla terra per sempre." L'essere dunque questo secolo "eminemment religiuex, cioè spiritualista" è una di quelle "follie" di cui le menti moderne soffrono, una di quelle follie partorite "non mica dall'ignoranza ma dalla scienza." Scosso dalla sua scoperta, Leopardi conclude l'attacco contro lo spiritualismo del suo tempo con un fitto contrasto tra la saggezza di coloro che sono immuni dal sapere, i fanciulli, i selvaggi e gli antichi, e la stoltezza di coloro che sanno, gli spiritualisti, i moderni: "L'idea chimerica dello spirito non è nel capo né di un bambino né di un puro selvaggio. Questi non sono spiritualisti, perché sono pienamente ignoranti. E i bambini, e i selvaggi puri, e i pienamente ignoranti sono per conseguenza a 
mille doppi piú savi de' piú dotti uomini di questo secolo de' lumi; come gli antichi erano piú savi quanto piú antichi, perché tanto piú ignoranti" (Zibaldone II 1035-36). ${ }^{6}$

Queste conclusioni chiudono il cerchio del pensiero leopardiano nello Zibaldone ricollegandosi alle considerazioni del 1820 dove il sapere, origine della perfettibilità e dell'immortalità, era condannato. Ma la credenza in un destino tanto grandioso quanto impossibile non solo mostra la pericolosa capacità d'illudersi del genere umano, ma anche la sua superbia. E la superbia allaccia il pensiero leopardiano a La ginestra, dove il sapere, la perfettibilità, l'immortalità e la nullità della vita trovano ultima, compiuta espressione e ripudio. Già nel dicembre 1820 Leopardi aveva definito la malefica illusione di grandezza impossibile "peccato di superbia" germogliato sul sapere e sulla ragione. Nel giro di sole quindici righe il poeta usava quest'espressione ben cinque volte: “Questo e non altro fu il peccato di superbia che gli scrittori sacri rimproverano ai nostri primi padri; peccato di superbia nell'aver voluto sapere quel che non dovevano ... peccato di superbia che a me pare che sia rinnovato precisamente da chi sostiene la perfettibilità dell'uomo. I primi padri ... peccarono appunto per aver sognata questa perfettibilità, e cercata questa perfezione fattizia. . . . Il loro peccato, la loro superbia, non consiste in altro che nella ragione. . . O Or questo appunto fu peccato e superbia" (Zibaldone I 338-39).

E Lcopardi oppone a questa superbia, parto del mendace sapere, il "verace saper" (La ginestra: 151), il sapere che è virtú e saggezza; oppone alla grandezza che è illusione malefica la "piccolezza" che non è umiltà, ma fede nel valore della vita. In una pagina dello $\mathrm{Zi}$ baldone dell'agosto 1823, Leopardi definisce l'individuo dotato "di maggiore e piú alto e piú capace intelletto e ingegno," cioè l'uomo ideale. Qucsti è colui che sa quale sia il suo posto nella vastità dell'universo, che sa i limiti del proprio essere e che conosce "la sua piccolezza." La conoscenza della "piccolezza" dimostra, non l'insignificanza dell'uomo, ma la sua "nobilità" e la "grandezza e potenza” del suo intelletto. Quando quest'uomo, davanti all'immensità dell'universo, "si sente essere infinitesima parte di un globo ch'è minima parte d'uno degl'infiniti sistemi che compongono il mondo, e in questa considerazione stupisce della sua piccolezza, e profondamente sentendola ... si confonde quasi col nulla, e perde quasi 
se stesso nel pensiero della immensità delle cose . . allora con questo atto e con questo pensicro egli dà la maggior prova possibile della sua nobilita, della forza e della immensa capacità della sua mente, la quale, rinchiusa in sí piccolo e menomo essere, è potuta pervenire a conoscere e intender cose tanto superiori alla natura di lui" (Zibaldone II 301-302). Quando, pochi anni avanti, nell'Infinito Leopardi confrontava gli "interminati spazi" e "l'infinito silenzio" dell'universo, "per poco il cor" del poeta "non si spaura" e, nella sua piccolezza, il suo pensicro s'annegava in "questa immensità," abbracciava la morte ("il naufragar"). Qui, nello Zibaldone, l'intreccio dei due centri del passo, "conoscere" e "piccolezza," genera invece un senso di vitalità, quello stesso senso di vitalità che la ginestra, nella sua inerme piccolezza, piú tardi mostrerà davanti al mondo ostile e minacciante in cui le è dato vivere.

La superbia era un tema della "psicologia sociale" del Settecento ed esprimeva l'ambizione dell'uomo ad esser quel che non era e non poteva essere- "innaturalmente buono, immoderatamente virtuoso e a vivere secondo ragione." Si sa che la superbia era condannata e che la condanna rifletteva l'aspirazione dell'epoca al primitivismo e all'anti-intellettualismo (Lovejoy 63-67). Leopardi condanna la superbia come il vizio della Restaurazione, di una generazione il cui corrotto sapere il poeta dileggia nella Palinodia al marchese Gino Capponi:

Oh menti, oh senno, oh sovrumano acume

dell'età, ch'or si volge! E che sicuro

filosofar, che sapienza, o Gino,

i piú sublimi ancora e piú riposti

subbietti insegni ai secoli futuri

il mio secolo e tuo! (208-213)

e ne I nuovi credenti ("Voi saggi, voi felici: anime clette / a goder delle cose," 103-104); di una generazione il cui corrotto sapere ne La ginestra rende tutto un secolo "superbo e sciocco" (53). Ne La ginestra il poeta assale il falso sapere che vuole l'uomo e la terra al centro dell'universo: "L'uomo non pur, ma questo / globo ove l'uomo c̀ nulla, / sconosciuto è del tutto" (172-74). Ed irride la superbia e l'errore dell'uomo: "Te signora c fine / credi tu data al Tutto" (188-89). Nell'ultimo canto il pocta ripudia la superba idea che "ciascheduno di noi ... si è tenuto per conto di essere uno imperatore; non mica di Constantinopoli o di Germania, ovvero della 
metà della terra . . . ma un imperatore dell'universo, un imperatore del sole, dei pianeti, di tutte le stelle visibili e non visibili" (Operette morali, "Il copernico, dialogo" 996-97), un idea che vive tenace nella mente retriva e reazionaria della Restaurazione, dell'epoca che segna il trionfo del potere ecclesiastico risorto in tutto la sua crudezza e arroganza sotto le ali tutelari della Santa Alleanza.

Il falso idolo della perfettiblità, "dell'umana gente / le magnifiche sorti e progressive" (50-51), indice supremo di superbia, diviene il bersaglio della critica leopardiana nell'ultimo canto. Si dimentica spesso che l'espressione "magnifiche sorti e progressive" è tratta da un'opera del cugino di Leopardi, Terenzio Mamiani, uno scrittorello pesarese ligio alla religione, fiducioso nella sua "virtú educatrice" e convinto che "la vita civile comincia dalla religione" (Gallo-Garboli 276, Straccali 300-301). Dimenticando questo, certa critica è pervenuta alla conclusione che la leopardiana demolizione delle magnifiche sorti e progressive scarta "qualsiasi ipotesi di storia, intesa come costruzione umana" (Botti 84). Le magnifiche sorti e progressive che Lcopardi demolisce ne La ginestra sono le sorti a cui aspira la Restaurazione, il movimento che faceva della religione uno strumento di governo, che potenziava il potere della nobiltà e della borghesia terriera, che avversava il libero pensiero e le aspirazioni nazionalistiche finché queste, soppresse ma non spente, sfoceranno nelle rivoluzioni di metà secolo. La perfettibilità che Leopardi rifiuta è quella tramata da una generazione retriva e violenta, una generazione che, volendo "servo . . . . . . il pensiero / sol per cui risorgemmo / dalla barbaric in parte, c per cui solo / si cresce in civiltà" (72-76), ignora il "verace saper." Lo ignora perché, inneggiando alle magnifiche sorti progressive, "fin sopra gli astri il mortal grado estolle" (86); dà prova di straordinaria superbia e rifugge da un sapere che, l'autorevole commentatore Straccali scrive, "faccia note le condizioni dell'uomo ... quali esse sono veramente" (308); rifugge da una sapere che ripudia la perfettibilità. La critica della perfettibilità ne $L a$ ginestra significa, non il rifiuto del progresso umano, ma il ripudio del movimento-la Restaurazione-che del progresso era fanatico nemico.

Come l'idea di perfettibilità, cosí anche altre correnti poetiche e intellettuali confluiscono ne La ginestra per acquictarsi nella certezza della verità ritrovata. Prima fra queste, la vanità della vita. Questa 
corrente è rappresentata dai versi $148-201$ in cui, dopo aver spaziato sulla piccolezza dell'universo e dell'umanità in esso, il poeta schernisce e compatisce l'uomo che, a dispetto della sua meschinità, "fin sopra gli astri il mortal grado estolle":

\section{... qual moto allora, \\ mortal prole infelice, o qual pensiero \\ verso te finalmente il cor m assale?}

Non so se il riso o la pietà prevale. (198-201)

In questo blocco di versi la critica ha, da una parte, scorto la "nullità dell'uomo" (Botti 74), e dall'altra una "visione integralmente relativistica," che comporta "un sostanziale appiattimento di ogni ipotetico valore" (Manacorda 189, 192). Un duplice malinteso vizia queste conclusioni. In primo luogo esse ignorano il fiore del deserto stesso, il protagonista del canto. Esso vive impavido davanti all'incessante, mortale minaccia del vulcano e rappresenta la "nobil natura ... / che grande e forte / mostra sé nel soffrir" (118-19). Per vivere impavido davanti allo spettro della morte, il fiore del deserto deve possedere una vitalità eccezionale; e questa mal s'accorda con il sentimento della nullità della vita. In secondo luogo quelle conclusioni sono viziate dalla mancata identificazione del soggetto per cui la vita è vana. Alla prima obiezione ritorneremo piú avanti. Quanto alla seconda, bisogna precisare che il soggetto dei versi 158-201 dell'ultimo canto non può essere la ginestra. Questa non si lamenta dell'esistenza e vive, finché la natura glielo permetterà, "contenta dei serti" allegrando con la sua presenza il desolato paesaggio che la circonda. La ginestra, "d'alma generoso e alto," è il "magnanimo animale" da cui emana un sentimento della vita opposto a quello della vanità. La nullità dell'uomo e l'appiattimento di ogni valore intravisti dalla critica nei versi 158-201 devono dunque riferirsi ad altri che alla ginestra. L'uomo per cui la vita è vana è colui che "di fetido orgoglio / empic le carte," che farnetica di "eccelsi fati e nove / felicità," e che "fin sopra gli astri il mortal grado estolle" $(86,88$, 102-104). È l'uomo della Restaurazione che, abbandonato "il risorto pensier segnato innanti," riabbraccia "i derisi sogni" dello spiritualismo, disdegna il "verace saper" e si nutre di "superbe fole." È colui che, per dirla ancora con Straccali, ha fatte sue quelle "credenze religiose per le quali l'uomo ... si reputa superbamente signore e fine dell'universo, e cura particolare di un Dio che gli destina una 
vita futura" (308-309). La vita è vana per colui il cui sapere nutre "l'aspettativa" in una vita immortale e felice nell'al di là.

Ma l'uomo libero dalle "superbe fole" "tende sempre alla vita" vissuta su questa terra perché, come Leopardi aveva già scritto altrove dei primitivi e degli antichi, cioè di color la cui vita non cessa mai di riscuotere la sua ammirazione, "la nullità, il non fare, il non viverc, è l'unica cosa di cui l'uomo sia incapace" (Zibaldone I 1245). A questo prepotente impulso vitale, c a quclla che entro qualche anno sarebbe divenuta l'ossatura poetica de La ginestra, Leopardi aveva alluso nclla celebre lettera del 1832 al De Sinner. Qui, cosciente del peso della vita, ma rifiutata la credenza in un al di là felice, il pocta riponeva la ragione di vivere nel fronteggiare l'avversità. "Quels que soient mes malherus," scriveva in maggio, “j’ai eu assez de courage pour ne pas chercher à en diminuer le poids ni par de frivoles espérances d'une prétendue félicité future et inconnue, ni par une lâche résignation." E con "ce même courage," Leopardi affermava d'esser pervenuto a "une philosophie désespérante," non per il gusto della disperazione, ma perché condottovi dallo spettacolo della vigliaccheria ("lâcheté") degli uomini (di coloro che ne $L a$ ginestra rifiuteranno il "risorto pensier segnato innanti" c abbracceranno le "superbe fole"), e perché credeva che quella sua filosofia, confrontando il vero nella sua nuda realtà, potesse ridare coraggio agli uomini, i quali “ont besoin d'être persuadés du mérite de l'existence" (Lettere 1033).

E come avrcbbe Leopardi potuto scrivere ne La ginestra i versi sulla "social catena" generata dal "verace saper" senza credere nel merito dell'esistenza? "L'umana compagnia," il poeta auspica,

tutti fra se confederati estima

gli uomini, e tutti abbraccia

con vero amor, porgendo

valida e pronta ed aspettando aita

negli alterni perigli e nelle angosce

della guerra comune. (129-35)

La novella solidarietà sociale avrebbe apportato "l'onesto $\mathrm{c}$ il retto / conversar cittadino, / e giustizia e pietade" tra gli uomini, e avrebbe rimpiazzato lc "supcrbe fole" a cui essi s'appigliano. Vero è che, come è stato detto, a causa della loro indeterminatezza i versi sulla socictà mancano di creare un'idea articolata e precisa sulla funzione della socictà di cui Leopardi scrive nell'ultimo canto (Timpanaro 
172, Bosco 89). Ma questa mancanza non esclude la leopardiana fede nel "merito dell'esistenza" e nell'impegno d'acerescerne il benessere. Questo impegno egli aveva già sostenuto nel $1820 \mathrm{am}$ monendo sull'insida che la dottrina cristiana rappresenta per la vita individuale e collettiva: il "Cristianesimo ... viene . . . a distruggere il mondo, la vita stessa individuale . . e sopratutto la società" ( $Z i$ baldone 1 1092). Questo stesso impegno sosterrà qualche anno dopo nel Discorso sopra lo stato presente dei costumi degl'italiani (1824) scrivendo che in società gli uomini possono "fare una tal quale stima della vita e delle cose umane" e lamentando la mancanza di società tra gl'italiani "di mondo," una mancanza che fa loro sentire "la vanità delle cose umane e della vita" (Le poesie e le prose II 565, 569). Se è vero che, come un critico ha di recente scritto, la ginestra "si alza dalla rovina" per assumere "il valore del sorgere della poesia stessa" e per segnalare "possibilità di verità e schiarita," questo accade non perché, come vuole il critico, "il silenzio dei deserti" c la rovina generano, come per incanto, il suo "contraltrare" (Spedicato 99). La rinascita avviene perché nella ginestra vive quella vita di cui il "secol superbo e sciocco," i credenti nelle "superbe fole" e l' "astuto e folle" che "fin sopra gli astri il mortal grado estolle" mancano. E questa vitalità (Iris Origo ha giustamente definito la ginestra "simbolo d'invicibile vitalità" [248]) permette alla ginestra di erigersi sopra la rovina circostante.

Ma niente meglio della rappresentazione dell'immortalità attesta il rifiuto della vanità della vita ne La ginestra. Alla fine del blocco dei versi che descrivono l'azione distruttrice del Vesuvio (237-96), dalla scomparsa di Pompei fino al presente, quando gli abitatori delle contrade ancora minacciati dal "flutto rovente / . . . della funerea lava," fuggono in preda al terrore, il poeta scrive: "Caggiono i regni intanto, / passan genti e linguaggi: clla [natura] nol vede: / c l'uom d'eternità s'arroga il vanto" (294-96). L'uomo si sente inerme e, davanti alla schiacciante forza della natura, supplisce alla sua impotenza con un atto d'arroganza: si fabbrica l'illusione dell'immortalità. D'altra parte, il canto si chiude con questi altri versi:

Ma piú saggia, ma tanto meno inferma dell'uom, quanto le frali tue stirpi non credesti o del fato o da te fatte immortali. (314-317) 
Qui la ginestra, pur essa soggetta alla forza distruttrice del vulcano come le cose e gli uomini del precedente blocco di versi, ripudia l'immortalità. L'ha ripudiata perché la ginestra ha osato vivere "contenta" e senza illusioni davanti all'avversità. Ed essa perirà senza né il terrore né il "fedito orgoglio" di colui che "fin sopra gli astri il mortal grado estolle"; perirà come se la morte non la toccasse:

.... E piegherai

sotto il fascio mortal non renitente

il tuo capo innocente

ma non piegato insino allora indarno

codardamente supplicando innanzi

al futuro oppressor; ma non eretto

con forsennato orgoglio inver le stelle. (304-310)

La fine della ginestra refuta l'idea che l'ultimo canto rappresenta l'inesorabile passare del tutto, il soccombere della "storia . . . alla natura" e l'accanita "corrosione di ogni progetto storico" (Manacorda 142, Botti 84). Il punto non è che tutto passa, che la storia soccombe; il punto è che, davanti alle vicende terrene, c'è una scelta da fare: vivere come l'uomo del "secol superbo e sciocco," o vivere come la ginestra. Nella sua superbia, il primo disprezza la vita mortale, "il vero / dell'aspra sorte e del depresso loco / che natura ci diè” (7880 ), e crede in una vita futura e migliore; nella sua saggezza e con il suo senso di solidarietà umana ("contenta dei deserti," commisera i "danni altrui," "il deserto consola" ed è "meno inferma dell'uom," $7,35,37,315)$, la ginestra ripone valore in una pur ardua esistenza e ripudia l'immortalità. L'uomo del "secol superbo e sciocco" incarna la verità che il poeta aveva intuito nel lontano 1820 quando meditanto sul Cristianesimo, intravide ch'esso induce a considerare "questa terra come un esilio" e a non aver cura "se non di una patria situata nell'altro mondo," e capí che il Cristianesmo "ha contribuito non poco a distruggere il bello il grande il vivo il vario di questo mondo" (Zibaldone I 248). La ginestra incarna la verità opposta: il bello, il grande, il vivo, il vario di questo mondo esistono se questa terra e questa vita sono considerate come la realtà, e se l'idea di una patria situata nell'altro mondo è ripudiata. La credenza nell'immortalità svaluta la vita senza liberarla dal timore della morte; l'accettazione della mortalità conferisce valore alla vita e la libera dal pensiero della morte.

Il ripudio della vanità della vita o, viceversa, l'affermazione del 
valore della vita, riveste un'importanzal speciale nell'ultimo canto. Per due ragioni. Primo, esso refuta decisivamente la dottrina dell'immortalità, una dottrina contro la quale, s'è visto, Leopardi s'oppose con accanito impegno lungo l'arco di tutto il suo pensiero. Secondo, il ripudio della vanità della vita corrobora la fede nella resurrezione o, se si vuole, nella perennità della vita umana. Se è vero che la ginestra, ̀̀ "simbolo d'invincibile vitalità," solo la fede nella resurrezione può esprimere adeguatamente questa qualità eccezionale. E solo un simbolo come la ginestra può rappresentare pocticamente questa fede. Il poeta immagina la ginestra, già all'inizio del canto, fiorente sulle millenaric rovine romane:

... Anco ti vidi

de' tuoi steli abbellir l'erme contrade

che cingon la citade

la qual fu donna de'mortali un tempo, (7-10)

e il lettore l'immagina risorgente durante i mille e ottocento anni che sono seguiti alla distruzione di Pompei. La ginestra che Leopardi contempla e gl'ispira l'ultimo canto non è il primo arbusto a comparire sulle pendici del Vesuvio. La "lenta ginestra" è forse perita ad ogni eruzione che versava il flutto di lava giú per il versante vulcanico. Ma nel corso dei secoli essa è cento volte risorta sul "flutto indurata" nella gloria dei suoi tralci verdeggianti e del suo giallo fiore, testimonio di vita imperitura.

Un'ultima osservazione che riconduce al punto di partenza, il sapere. La vita e la morte esemplare della ginestra sono sostenute da qualcosa piú potente di quel che la critica le attribuisce, la "cognizione del dolore" (Botti 77). Quella piccolezza che nello Zibaldone il pocta aveva chiamato grandezza, rivive ne La ginestra. Qui il fiore del deserto, materialmente piccolo dinnanzi alla strapotenza della natura, diviene la "nobil natura . . . che grande e forte / mostra sé nel soffrir." Quella piccolezza che consegna l'uomo di Pascal nelle mani misericordiose di Dio (l'uomo è grande perché riconosce la sua miseria e si rimette a Dio [Pascal 131]), diviene forza nella ginestra. Questa piccolezza che genera nel pigmeo la forza del gigante sorge dal "sapere," da un sapere al quale il poeta attribuisce un valore speciale. Si ricorderà che nello Zibaldone il sentimento della piccolezza-grandezza nasceva solo nell'individuo dotato "di maggiore e piú alto e piú capace intelletto ed ingegno" (Zibaldo- 
ne II 301-302). Quell'eccezionale intelletto s'incarna nella ginestra che, "piú saggia, ma tanto / meno inferma dell'uom," mostra un sapere superiore, la saggezza appunto. "The plant is the paragon of wisdom; it is wisdom" (Nelson, Jr., 357), un critico ha detto; e la Saggezza è la quintessenza del sapere. Questo sapere, "il pensiero, / sol per cui risorgemmo / dalla barbarie in parte," dalla barbarie dello spiritualismo e delle "superbe fole," è il "verace saper" che cimenta la solidarietà sociale, la prerogativa della "nobil natura" e del "magnanimo animal." È il sapere che s'oppone a quello mendace della Restaurazione, nemico del "risorto pensier segnato innanti." "E servo . . . / vuoi," il poeta redarguisce il "secol superbo e sciocco," "di novo il pensiero / sol per cui risorgemmo." È il sapere che, nella sua suprema manifestazione, ripudia l'immortalità:

Ma piú saggia, ma tanto

meno inferma dell'uom, quanto le frali

tue stirpi non credesti

o del fato o da te fatte immortali.

Se queste considerazioni non sono errate, esse refutano l'ipotesi che La ginestra esprime un "pessimismo integrale" (Manacorda 104) e confermano, invece l'intuizione di Francesco De Sanctis che, leggendo Leopardi, "ti senti stringere piú saldamente a tutto ciò che nella vita è" vitale (466), un'intuizione che doveva restare tale perché lo Zibaldone, la chiave di volta del sistema leopardiano, rimase sconosciuto al critico irpino.

University of California, Santa Barbara

\section{NOTE}

1 Questo corsivo, come quelli che seguono, sono di Leopardi.

2 Le lettere cubitali sono di Leopardi.

3 Poco studiata la religione in Leopardi. Ma v. Porena, che traccia il "distaccarsi" del poeta dalla religione e la formazione di una "concezione universalmente pessimista"; Timpanaro 196, scrive della "ripugnanza" di Leopardi verso le idee cristiane, "illusioni negatrici di vita attiva, di felicità terrena"; Bonadeo, "Leopardi e la religione" 37-48.

4 Sul corpo e lo spirito in Leopardi, v. Bonadeo, "Il corpo e il vigore nello Zibaldone di Leopardi" 55-65.

5 Sull'Inno e sull'età dell'oro come "ignoranza," v. Costa 221-22. 
6 Queste riflessioni del poeta bastano a refutare certa critica che ha visto l'anima di Leopardi "sitibonda" di luce spirituale e sollevata ai "regni dell'inifinito": Frattini, 127.

\section{OPERE CONSULTATE}

BONADEO, Alfredo. "Il corpo e il vigore nello Zibaldone di Leopardi." Italianistica 5 (1976): 55-65.

“"Lcopardi e la religione." Italian Quarterly 25 (1984): 37-48.

BOSCO, Umberto. Tiranismo e pietà in Giacomo Leopardi. Roma: De Santis, n.d.

BOTTI, Francesco. "La ginestra: flessioni e contraddizioni di un simbolo." Modern Language Notes 90 (1975).

COSTA, Gustavo. La leggenda dei secoli d'oro nella letteratura italiana. Bari: Laterza, 1972.

DE SANCTIS, Francesco. Leopardi. Ed. Muscetta-Perna. Torino: Einaudi, 1969.

FRATTINI, Alberto. "Leopardi e il problema religioso." In Studi leopardiani. Pisa: Nistri Lischi, 1956.

LEOPARDI, Giacomo. Lettere. In Tutte le opere. Ed. F. Flora. Milano: Mondadori, 1961.

- Zibaldone di pensieri. In Tutte le opere.

. Operette morali. In Tutte le opere: Le poesie e le prose I. Ed. F. Flora. Milano: Mondadori, 1958.

- Comparazione delle sentenze di Bruto Minore e di Teofrasto vicini a morte. In Le poesie e le prose 1.

- Argomenti e abbozzi di prose: "Inno ai Patriarchi o de" principii del genere umano." In Le poesie e le prose I.

. Paralipomeni della batracomiomachia.

"Il copernico, dialogo." Operette morali. In Le poesie e le prose I.

Canti. Con commenti di N. Gallo-C. Garboli. Torino: Einaudi, 1972.

Canti. Con commenti di Alfredo Straccali. Firenze: Sansoni, 1962.

LOVEJOY, Arthur. "Pride in Eighteenth-Century Thought." In Essays in the History of Ideas. New York: Putnam's Sons, 1960.

MANACORDA, Giorgio. Materialismo e masochismo. Il Werther, Foscolo e Leopardi. Firenze: La Nuova Italia, 1973.

NELSON, Jr., Lowry. "Leopardi First and Last." In Italian Literature: Roots and Branches. Ed. Rimanelli-Atchity. New Haven: Yale UP, 1976.

ORIGO, Iris. Leopardi, a Study in Solitude. Londra: Hamilton, 1953.

PASCAL, Blaise. Pensées. In Pensées and the Provincial Letters. New York: The Modern Library, 1941.

PORENA, Manfredi. Scritti leopardiani. Bologna: Zanichelli, 1959.

SPEDICATO, Paolo. "Elogio dell'upupa: rovine della letteratura e rovine del 
pensiero." Modern Language Notes 101 (1986).

TIMPANARO, Sebastiano. Classicismo e Illuminisimo nell'Ottocento italiano. Pisa: Nistri Lischi, 1965. 